\title{
Mapping Evidence on Global Perceptions of Environmental Sustainability amidst the COVID-19 Pandemic: A Systematic Review Protocol
}

PETER ANSU-MENSAH ( $\nabla$ peter.ansumensah@ymail.com )

Sunyani Technical University https://orcid.org/0000-0002-3961-3109

Monica Ansu-Mensah

Sunyani Technical University

Desmond Kuupiel

University of Kwazulu-Natal

\section{Research}

Keywords: COVID-19, Pandemic, Environmental Sustainability, Perception, Worldwide

Posted Date: January 19th, 2021

DOl: https://doi.org/10.21203/rs.3.rs-148450/v1

License: (c) (i) This work is licensed under a Creative Commons Attribution 4.0 International License.

Read Full License 


\section{Abstract}

Background: Identifying and addressing research gaps on environmental sustainability in this striving time of COVID-19, it is imperative to ensure proper waste management disposal, efficient use of energy as well as judicious use of transport in order to achieve the SDGs 2030.

Aim: The proposed scoping review is aimed at mapping evidence on global perceptions of environmental sustainability amidst COVID-19 pandemic.

Methods: The proposed study will be guided by the enhanced version of Arksey and O'Malley's scoping review framework, and Levac et al. 2010 recommendations together with the 2015 Joanna Briggs Institute guidelines. A comprehensive keywords search for relevant studies presenting evidence of environmental sustainability during COVID-19 pandemic will be conducted with the following databases: SCOPUS, Google Scholar, EBSCOhost, and PubMed. Literature from university repositories and international organization such as the World Health Organization (WHO) and government websites relevant to the proposed study will also be retrieved. The proposed review will use the Preferred Reporting Items for Systematic Reviews and Meta-analysis: Extension for Scoping Review (PRISMA-ScR) to present the results of the study. For data extraction in a content thematic manner of analysis, NVivo version 11 software package will be used. A mixed methods appraisal tool (MMAT) version 2018 will be employed to appraise the quality of all the included studies.

Discussion: We are hopeful that the results of the proposed study will inform future research and unveil evidence-based information to report potential environmental sustainability issues that may arise in this new phase of COVID-19 pandemic. It also anticipated that the proposed study will enable policy and regulatory bodies to implement new strategies to achieve the SDGs inclusive of COVID-19 pandemic.

\section{Introduction}

The United Nations (UN) basically initiated the seventeen (17) Sustainable Development Goals (SDGs) 2030 with the aim of improving the quality of life around the globe (1). One of its scope is environmental which is interconnected in many of the SDGs for the benefit of improving global resource consumption and production and thereby achieving sustainable environment (2). It is worth noting that almost $50 \%$ of the SDGs, seek to achieve sustainable environment worldwide either through direct or indirect means (3, 4). The presence of Coronavirus disease (COVID-19) pandemic has caused a challenge on the plans made for attaining these goals through the slowdown of socio-economic development which if not resolved would pose a serious threat on the said goals (5). Again, lockdown restrictions on industrial activities and transportation have resulted in an increase in pollutant concentration and a reverse of indoor air quality (6). Also, as movements of people are restricted indoors, there is greater use of energy especially electricity among individuals and households worldwide $(7,8)$ In this regard, sustainable environmental management plan would be relevant in order to achieve the related SDGs (9). 
Nonetheless, the COVID-19 movement restrictions such as the lockdown have influenced the environment in a positive and negative manner. For instance, in a constructive manner, there is no excess carbon emission and water pollution in many cities globally (10). Similarly, the adoption of behavioural and structural changes regarding demands for fossil fuel and support for climate change modification amidst the COVID-19 pandemic create greater chance of green stimulus paving way for sustainable environment (8). The impact of demand and supply of electricity during the pandemic vary countywide; from decline in demand to no effect comparatively in some European countries (11). Furthermore, the reduction in energy consumption and pollution from industrial activities has impacted positively on the environment which consequently has enhanced sustainability (12). Large volume of air flights and motor vehicles declined due to industrial lockdown and this would certainly reduce the amount of pollution and emission of greenhouse gases (GHG) (13). In Brazil, the amount of street waste as well as domestic solid waste decreased as compared to the pre-COVID-19 era (14). Likewise, in North America, the volume of solid waste has drastically reduced owing to the lockdown restrictions (15).

On the other hand, environmental issues such as pollution policies, regulation, and waste recycling had not been attended to amidst the pandemic (13). Equally, the presence of the COVID-19 pandemic would undermine the progress of low-income economies like Ghana. Thus, international sources of funds, such as loans and grants needed to carry out interventions to achieve SDGs 2030 would be difficult to acquire (16). Consequently, the production and manufacturing of COVID-19 Personal Protective Equipment (PPEs) including nose masks, gloves, and disposable overalls hamper the environment (17).

Besides, due to urbanization, the spread of infectious diseases have become eminent because of high concentration of people in smaller areas, coupled with poor sanitary conditions, and no interventions to alleviate risks of infection (18). Though mostly affected COVID-19 people are those with underlining medical conditions, poor environmental conditions expose the minority group to higher risks (19). Therefore, it is needful for schools around the globe to include environmental hygiene in their curriculums in order to forestall future spread of the outbreak of infectious diseases like COVID-19 (20).

This study intends to map evidence of environmental sustainability amidst the COVID-19 pandemic and to find out research gaps in the research area of interest. It is also anticipated that this study's result will make known new policies and regulations that can be used to address the current myriad of problems related to environmental sustainability. Besides, the new policies that have come about due to COVID-19 are needed so as to achieve the SDGs 2030. Similarly, it will aid in resolving how to overcome these harms in order to avert the degradation of the environment in this testing times of COVID-19.

\section{Methods}

\section{Overview}

Scoping review will map range of literature, which exist around this research field of interest and ascertain study gaps for posterity $(21,22)$. Scoping methodology is considered the most useful approach 
for determining need and value of systematic review or primary study (21). As a result, this study will be guided by the 2005 Arksey and O'Malley's scoping review framework, Leva et al. 2010 recommendations $(21,23)$ specifying the following: research questions identification; identification of relevant; study selection; charting and collation of data, summarising and reporting findings using the 2015 Joanna Briggs Institute Guidelines (24). This study protocol is being reported in line with the Preferred Reporting Items for Systematic Reviews and Meta-Analyses Protocol (PRISMA-P) account $(25,26)$.

\section{Identifying the research question}

Our research question is: What is the evidence of environmental sustainability during the COVID-19 pandemic across the globe?

The sub-review questions will be as follows:

- What waste management practices contributed to environmental sustainability around the world during the COVID-19 pandemic?

- What industrial activities influenced the transmission or spread of the COVID-19 pandemic?

- What kind of environmental hazard existed, and how did it affect the outbreak of the COVID-19 pandemic?

- What are the implications on the global environment because of the closure of commercial activities due to lockdowns?

The Population, Exposure and Outcome (PEO) mnemonic (27) will be used to determine the eligibility of the proposed scoping review question as shown in Table 1.

Table 1

PEO framework for defining the eligibility of the studies for the primary research question

\begin{tabular}{|ll|}
\hline P: Population & Children and Adults. \\
\hline E: Exposure & Domestic waste, Industrial waste, Energy waste, Transport waste. \\
& Environmental pollution \\
& Water pollution \\
& Depletion of natural resources \\
& Increase in global warming \\
& Increase in GHG emissions \\
Identify relevant studies
\end{tabular}

A complete and thorough search of numerous bibliographic databases will be conducted to identify all relevant studies on the global perceptions of environmental sustainability amidst the COVID-19 pandemic 
regardless of publication status (published, unpublished or press documents). Additional studies from university repositories, government, and international organizations websites will be included in searching for relevant studies. We will use the following databases: SCOPUS, Google Scholar, Science Direct EBSCOhost (Academic Search Complete), and PubMed to retrieve all relevant studies. Combination of search terms and appropriate keywords such as "children" OR "child" OR "kid" OR "youngers" OR "teenagers" OR "adolescent" OR "youth" OR AND "adults" OR "grown-ups" AND "environmental pollution" "water pollution" OR "burden" AND "pandemic" "outbreak" "covid-19" "transport waste" "energy waste" "domestic waste" "industrial waste" "worldwide" OR "global" "across the globe" will be used. Boolean terms (AND and OR) and Medical Subject Heading (MeSH) will be used to separate keywords. Date of search, type of database, number of identified studies and number of eligible studies will be indicated as shown in Table 2.

Table 2

Pilot search in PubMed electronic database

\begin{tabular}{|c|c|c|c|}
\hline Date & Database & Keywords & $\begin{array}{l}\text { Search } \\
\text { results }\end{array}$ \\
\hline $31 / 12 / 2020$ & PubMed & $\begin{array}{l}\text { ((((""global"[All Fields] AND ("perception"[MeSH Terms] OR } \\
\text { "perception"[All Fields] OR "perceptions"[All Fields])) AND } \\
\text { ("environment"[MeSH Terms] OR "environment"[All Fields] OR } \\
\text { "environmental"[All Fields]) AND "sustainability"[All Fields])) } \\
\text { OR ("waste management"[MeSH Terms] OR ("waste"[All Fields] } \\
\text { AND "management"[All Fields]) OR "waste management"[All } \\
\text { Fields])) OR ("energy"[All Fields] AND conservation[All Fields])) } \\
\text { OR (("biological transport"[MeSH Terms] OR ("biological"[All } \\
\text { Fields] AND "transport"[All Fields]) OR "biological transport"[All } \\
\text { Fields] OR "transport"[All Fields]) AND ("waste management" } \\
\text { [MeSH Terms] OR ("waste"[All Fields] AND "management"[All } \\
\text { Fields]) OR "waste managenent"[All Fields]))) AND ("covid-19" } \\
\text { [MeSH Terms] OR "covid-19"[All Fields] OR "covid 19 } \\
\text { pandemic"[All Fields]) }\end{array}$ & 3774 \\
\hline
\end{tabular}

\section{Eligibility criteria and study selection}

The study selection will be guided by eligibility criteria specifically under inclusion and exclusion for easy retrieval of relevant studies.

\section{Inclusion criteria}

Studies meeting the following criteria will be included:

- Evidence of the study conducted anywhere in the world

- Evidence of the study conducted during COVID-19

- Studies presenting evidence of domestic waste management amidst COVID-19

- Studies reporting evidence of industrial waste management amidst COVID-19

- Studies presenting evidence of energy conservation during COVID-19 pandemic 
- Studies presenting evidence of environmental sustainability in the COVID-19 era

- Quantitative, qualitative, and mix methods study designs

\section{Exclusion criteria}

This study will exclude the following:

- Studies presenting evidence of environmental sustainability but published before COVID-19 pandemic

- Studies relating to COVID-19 but do not report on environmental sustainability

- Other types of reviews

\section{Study selection}

Screening of the proposed study will be conducted in three phases to select relevant studies. In the phase, the principal reviewer will conduct the title screening using the various online databases. All relevant studies will be imported onto Mendeley desktop created for this study. Secondly, two independent trained investigators in the classifications of inclusive and exclusive study using the eligibility criteria will conduct abstract and full-text screening stages respectfully. A third reviewer in a discussion manner will resolve all discrepancies that may arise between the two investigators at the abstract screening stage until agreement is reached. In situation where a full-text article cannot be accessed, assistance would be sought from the Sunyani Technical University's Library or by writing to the author to provide it through email. Inter-rater agreement (Cohen's kappa co-efficient ( $k$ ) statistics) between reviews' responses will be calculated as well as the McNemar's chi-square statistics using Stata 14 following full text screening. Details of search records including date of search, database, keywords, number of studies identified, and the number of eligible studies will be appropriately recorded. The authors will follow an adapted "Preferred Reporting Items for Systematic Reviews and Meta-analysis" (PRISMA) guideline to report the screening result (26) as shown in Fig. 1.

\section{Charting the data}

For easy retrieval of data by all reviewers, data charting form will be designed using excel spreadsheet for extraction of relevant data from the included studies in this scoping review. The form will include the following headings: author and date, study title, aim of study, study design, study setting, energy conservation practices, and waste management practices, environmental sustainability approaches. Pilot study to test for consistency and accuracy of data extraction form will be conducted by two independent reviewers using a random sample of 10 . The data form will be adjusted constantly based on feedback from both reviewers throughout the duration of the study.

\section{Collating, summarizing, and reporting the results}


We will extract data related to environmental sustainability in the COVID-19 era using content thematic approach. All finding from the included studies will be analyzed by the application of NVivo version 11 . Thematic content analysis on environmental sustainability amidst COVID-19 pandemic summarized and presented in a narrative approach as emerging themes reported

Table 3

Data extraction form

Author and publication date

Study title

Study aim

Type of study design

Study setting

Study population

Sample size

Study findings (energy waste, transport waste, industrial/domestic waste)

Significant finding

Remarks

\section{Quality appraisal}

Mixed Method Quality Appraisal Tool (MMAT) version 2018 will be employed to evaluate the methodological quality of all the included studies to ensure the quality of the proposed scoping review. Thorough examination will be conducted using the MMAT tool to assess the relevance of the study, adequacy and methodology, study design, data collection, analysis of data and study findings. This quality assessment will help report the risk of bias of the included studies and the overall quality of evidence that will be reported.

\section{Discussions}

This proposed study will aim to map evidence on global perceptions of environmental sustainability during the COVID-19 pandemic. Improving energy usage, transport and waste management have significant effect on the environment. Consequently, global health crises could be turned into opportunity if we are able to use the lockdown period to provide security to human being and the environment (8). Thus, conserving the water bodies, land, air and sea by engaging in proper waste management practices, as well as efficient use of energy, and transport and thereby achieve a sustainable environment. It is anticipated that the results of the proposed scoping review will inform researchers and disseminate information to the policy and regulatory bodies around the world; addressing potential environmental issues that may arise due to the COVID-19 outbreak. The study will also be useful to global world in 
planning and implementation of environmental issues; to factor any unforeseen destruction on the environment and the preparedness to overcome its challenges. Again, the study will enhance strict regulations of the measures to achieve sustainable environment.

\section{Conclusion}

The results of this systematic scoping study will provide evidence that will be beneficial to future research in the area of systematic reviews, primary studies and meta-analysis to influence policy and regulatory bodies in strategizing for sustainable environment.

\section{Abbreviations}

COVID-19 : Coronavirus Disease

GHG : Greenhouse Gases

MMAT : Mixed Method Quality Appraisal Tool

PEO : Population, Exposure, and Outcome

PPEs : Personal Protective Equipment

SDGs : Sustainable Development Goals

UN : United Nations

\section{Declarations}

\section{Acknowledgments}

We are appreciative to the Almighty God for the divine protection and guidance throughout the writing of this protocol study.

\section{Author's contribution}

PA-M and MA-M conceptualized the study. PA-M wrote the first draft as well as the writing of the protocol. MA-M and DK made the critical revision. PA-M wrote the final draft, approved by all authors.

\section{Funding}

This proposed study has not received any funding yet 


\section{Ethics approval and consent to participate}

Not applicable

\section{Consent to publish}

Not applicable

\section{Data availability}

We have appropriately cited all study and all data presented in the form of references

\section{Competing interests}

None declared

\section{References}

1. Workie E, Mackolil J, Nyika J, Ramadas S. Current Research in Environmental Sustainability Deciphering the impact of COVID-19 pandemic on food security, agriculture, and livelihoods: A review of the evidence from developing countries. Curr Res Environ Sustain. 2020;2:100014.

2. Fleetwood J. Social Justice, Food Loss, and the Sustainable Development Goals in the Era of COVID-19. 2020;

3. UNDP. Sustainable Development Goals. report. 2015;24.

4. General UN, Summit A, Development S, Goals SD, Sustainable T, Goals D, et al. No Title.

5. Ukherjee ABM, Abu SSUB, Hosh SUG. Thinking about water and air to attain Sustainable Development Goals during times of COVID-19 Pandemic. 2020;0123456789.

6. Somani M, Srivastava AN, Gummadivalli SK, Sharma A. Bioresource Technology Reports Indirect implications of COVID-19 towards sustainable environment: An investigation in Indian context. Bioresour Technol Reports. 2020;11:100491.

7. Shupler M, Mwitari J, Gohole A, Anderson de Cuevas R, Puzzolo E, Cukic I, et al. COVID-19 Lockdown in a Kenyan informal settlement: Impacts on ousehold energy and food ecurity. MedRxiv. 2020;

8. Kuzemko C, Bradshaw M, Bridge G, Goldthau A, Overland I, Scholten D, et al. Covid-19 and the politics of sustainable energy transitions. Energy Res Soc Sci. 2020;68(June):101685.

9. Bera B, Bhattacharjee S, Kumar P. Significant impacts of COVID - 19 lockdown on urban air pollution in Kolkata (India ) and amelioration of environmental health. Environ Dev Sustain. 2020; (0123456789). 
10. Nilashi PFRM, Asadi RAAS, Wang SSS. Coronavirus Pandemic (COVID - 19) and its Natural Environmental Impacts. Int J Environ Sci Technol. 2020;17(11):4655-66.

11. Kanda W, Kivimaa P. Energy Research \& Social Science What opportunities could the COVID-19 outbreak o ff er for sustainability transitions research on electricity and mobility? Energy Res Soc Sci [Internet]. 2020;68(May):101666. Available from: https://doi.org/10.1016/j.erss.2020.101666

12. Altieri MA, Nicholls Cl. Agroecology and the reconstruction of a post- COVID-19 agriculture. 2020;

13. Jones P, Comfort D. The COVID-19 crisis and sustainability in the hospitality industry. 2020;32(10):3037-50.

14. Custodio R, Yuri L, Nakada K. Science of the Total Environment COVID-19 pandemic: Solid waste and environmental impacts in Brazil. Sci Total Environ. 2021;755:142471.

15. Kulkarni BN, Anantharama V. Repercussions of COVID-19 pandemic on municipal solid waste management: Challenges and opportunities. Sci Total Environ. 2020;140693.

16. Barbier EB, Burgess JC. Sustainability and development after COVID-19. World Dev [Internet]. 2020;135:105082. Available from: https://doi.org/10.1016/j.worlddev.2020.105082

17. Zhang D, Hao M. Is Environmental Sustainability Taking a Backseat in China after COVID-19? The Perspective of Business Managers. 2020;

18. Pinheiro MD. COVID-19 Could Leverage a Sustainable Built Environment. 2020;

19. Sovacool BK, Furszyfer D, Rio D, Griffiths S. Energy Research \& Social Science Contextualizing the Covid-19 pandemic for a carbon-constrained world: Insights for sustainability transitions, energy justice, and research methodology. Energy Res Soc Sci. 2020;68(August):101701.

20. Toquero CM. Challenges and Opportunities for Higher Education amid the COVID- 19 Pandemic: The Philippine Context. 2020;5(4).

21. Arksey H, Malley LO, Arksey H, Malley LO. Scoping studies: towards a methodological framework Scoping Studies: Towards a Methodological Framework. 2007;5579.

22. Bhsc MDJP, Ma Q, Rn CMG, Bpharm HK, Mcinerney P, Parker D, et al. Guidance for conducting systematic scoping reviews. 2015;

23. Levac D, Colquhoun H, Brien KKO. Scoping studies: advancing the methodology. 2010;1-9.

24. Briggs J. Conducting systematic reviews of association (etiology): The Joanna Briggs Institute's approach. 2015;

25. Petticrew M, Shekelle P, Stewart LA, Group P. meta-analysis protocols (PRISMA-P) 2015 : elaboration. 2015;7647(January):1-25. Available from: http://dx.doi.org/doi:10.1136/bmj.g7647

26. Moher D, Shamseer L, Clarke M, Ghersi D, Liberati A, Petticrew M, et al. Preferred reporting items for systematic review and meta-analysis protocols (PRISMA-P) 2015 statement. 2015;1-9.

27. Danquah FI, Yeboah M, Bawontuo V, Kuupiel D. Mapping evidence on the burden and distribution of childhood obesity in sub- Saharan Africa: a scoping review protocol. 2019;4-9.

\section{Figures}




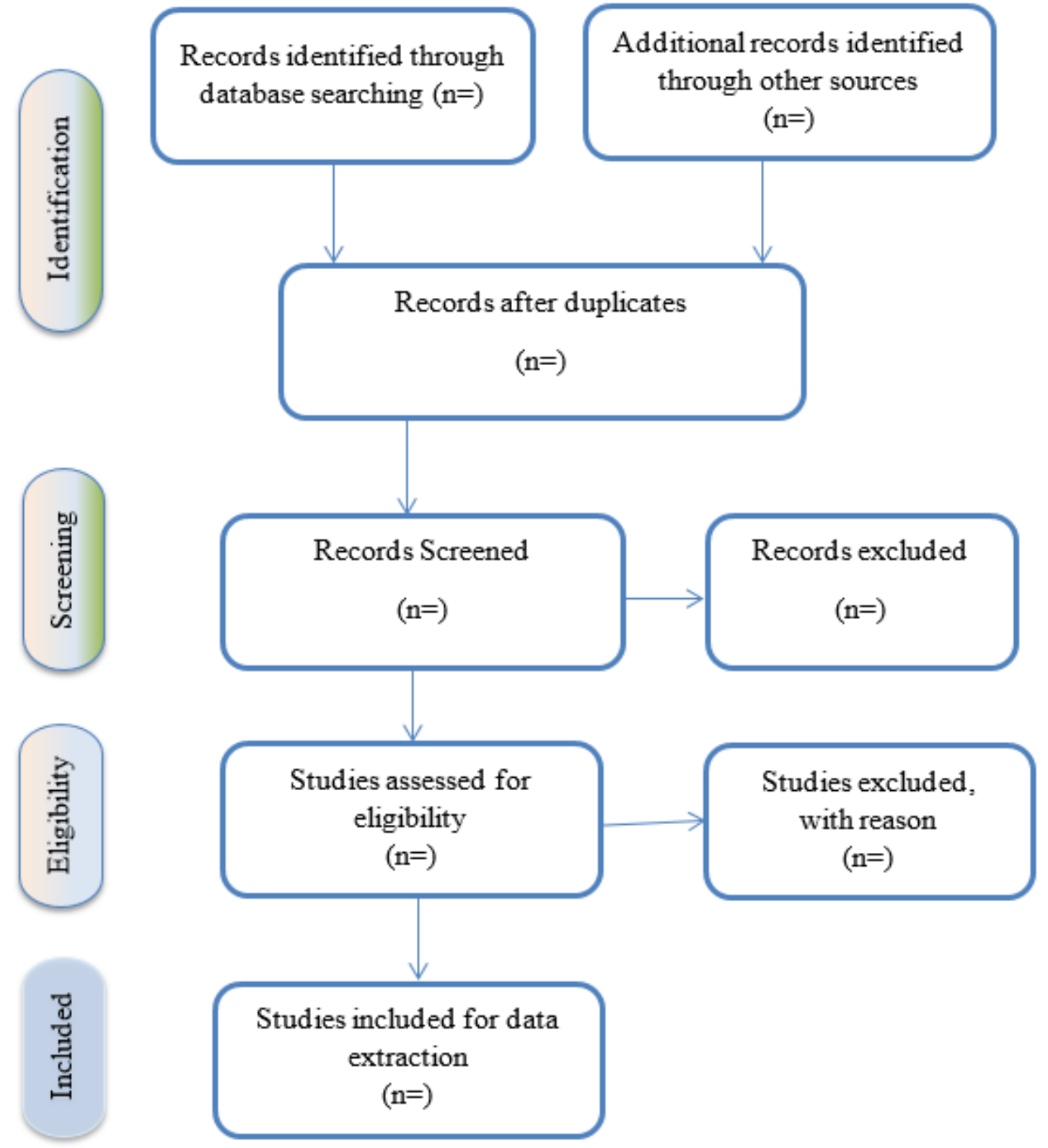

Figure 1

PRISMA 2009 Flow Diagram (26) 